\title{
Provisionamento Multicaminho com Otimização de Particionamento de Taxa de Transmissão para Proteção Dedicada em Redes Ópticas Elásticas
}

\author{
Henrique A. Dinarte, Daniel A. R. Chaves e Raul C. Almeida Jr, Karcius D. R. Assis e Helio Waldman.
}

\begin{abstract}
Resumo-Dentre os esquemas de proteção multicaminho encontrados na literatura, o esquema de proteção dedicado particionado (Partitioning Dedicated Path Protection - PDPP) propõe a partição da taxa de transmissão total demandada pela conexão em partes iguais e a transmissão por múltiplos caminhos disjuntos. Entretanto, é possível particionar tal taxa desigualmente entre os caminhos, permitindo benefícios para o desempenho da rede. Este artigo propõe uma meta-heurística que utiliza algoritmo genético para encontrar o melhor particionamento da taxa de transmissão total da conexão, de forma a prover redução na probabilidade de bloqueio e na na fração média de banda espremida da rede.
\end{abstract}

Palavras-Chave - proteção multicaminho, algoritmo genético, proteção dedicada com banda espremida, redes ópticas elásticas.

Abstract-Among the multipath protection schemes found in the literature, the Partitioning Dedicated Path Protection - PDPP proposes to divide the total transmission rate required by the connection in equal parts and then, proceed the transmission of each part using multiple disjoint paths. Nevertheless, it is also possible to partition the transmission rate unequally between paths, enabling benefits to network performance. This article proposes a meta-heuristic based on genetic algorithms to find an optmized partitioning of connections transmission rate among multiple disjoint paths, in order to provide dedicated protection and reduction in the blocking probability and average bandwidth squeezing.

Keywords-multipath protection, genetic algorithm, dedicated protection with bandwidth squeezing, elastic optical networks.

\section{INTRODUÇÃO}

Uma importante questão nas redes ópticas de transporte é a sua sobrevivência. Essas redes geralmente são utilizadas para transmitir serviços que requerem grandes volumes de tráfego e altas taxas de disponibilidade. Uma possível falha em um nó ou enlace da rede pode causar a interrupção dos serviços e gerar grande perda de dados e receita para a empresa prestadora do serviço de transporte de informação [1]. Um dos mecanismos para promover sobrevivência em uma rede óptica é o mecanismo de proteção. Mais especificamente, a proteção contra falha em enlace da rede consiste em encontrar um caminho principal e um caminho reserva, disjunto do primeiro,

Raul C. Almeida Jr, Departamento de Engenharia Eletrônica e de Sistemas, UFPE, Recife-PE, e-mail: raul.almeidajunior@ufpe.br; Henrique A. Dinarte, Programa de Pós-Graduação em Engenharia Elétrica, UFPE, Recife-PE, email: henrique.dinarte@ufpe.br; Daniel A. R. Chaves, Escola Politécnica de Pernambuco, UPE, Recife-PE, e-mail: darc@ecomp.poli.br; Karcius D. R. Assis, Departamento de Engenharia Elétrica e Computação, UFBA, Salvador-BA, e-mail: karcius.assis@ufba.br; Helio Waldman, Departamento de Engenharia de Comunicações, UNICAMP, Campinas-SP, e-mail: hwaldman@unicamp.br. e reservar esse par de recursos para cada conexão solicitada à rede que deve ser protegida.

Em redes ópticas elásticas (Elastic Optical Networks EON) [2], os esquemas de proteção, comumente, utilizam dois tipos de provisionamento: o provisionamento de caminhoúnico (Single-path Provisioning - SPP) e o provisionamento multicaminho (Multipath Provisioning - MPP). No primeiro, um caminho óptico de trabalho é protegido com um ou mais caminhos disjuntos de proteção. Entretanto, a taxa de transmissão requisitada $\left(B_{r}\right)$ não é particionada entre os caminhos. Assim, cada caminho de proteção possui uma taxa de transmissão incremental para prover a proteção $\left(B_{i}\right)$ igual à taxa de transmissão requisitada. $O$ esquema de proteção $1+1$ é um caso particular do SPP, no qual o caminho de trabalho é protegido com apenas um único caminho disjunto de proteção. Nesse caso, a taxa de transmissão total utilizada $\left(B_{t}\right)$ para estabelecer uma conexão protegida na rede consiste na soma das taxas de transmissão requisitada e incremental para proteção $\left(B_{r}+B_{i}\right)$. No caso da proteção $1+1, B_{t}$ corresponde ao dobro de $\left(B_{r}\right)$. O segundo tipo, o MPP, propõe o particionamento de $B_{r}$ em múltiplas parcelas de menores taxas de transmissão, ou taxas de transmissão parciais $\left(B_{s}\right)$, e a sua transmissão é feita de forma simultânea por múltiplos caminhos disjuntos. Diferentemente do SPP, no MPP não há distinção entre caminho óptico de trabalho e de proteção, visto que todos os caminhos ópticos envolvidos podem ser usados para transmissão de dados da conexão, seja na operação normal da rede ou sob falha em um dos enlaces das rotas.

A implementação do MPP é possível, principalmente devido ao desenvolvimento dos Sliceable Bandwidth-Variable Transponders - SBVTs, capazes de suportar a configuração de múltiplas subportadoras que se adaptam ao tráfego demandado e podem ser transmitidas por diferentes caminhos, graças à funcionalidade de fatiamento (sliceability) [3].

Assis et al. [4], [5] investigaram esquemas de proteção dedicada (Dedicated Path Protection - DPP) e propuseram um esquema de particionamento de tráfego chamado (Partitioning Dedicated Path Protection - PDPP). O DPP é um esquema do tipo SPP que realiza a proteção do tráfego de requisitado por meio de um único caminho de proteção para a mesma taxa de transmissão. O PDPP, por sua vez, propõe o particionamento de $B_{t}$ igualitariamente por caminhos disjuntos, com o objetivo de reduzir os recursos consumidos da rede em relação ao DPP. Os autores também abordam o conceito de squeezing que consiste na possibilidade de, sob a ocorrência de falha, a taxa de transmissão ser reduzida (espremida) em ralação a 
$B_{r}$. Tal conceito pode ser aplicado tanto ao DPP quanto ao PDPP.

Considerando uma rede óptica elástica de tráfego dinâmico, o percentual de squeezing ao qual uma chamada pode ser submetida e a probabilidade de bloqueio total da rede (PB) podem se vistos como parâmetros de otimização conflitantes entre si em uma EON. Isso porque o aumento do máximo squeezing tolerável faz as conexões demandarem menos largura de banda da rede, o que provoca uma redução da PB. A reciproca também se verifica.

Neste trabalho é feita uma adaptação dos esquemas de proteção para redes de tráfego estático, apresentados em [5], para redes de tráfego dinâmico. $\mathrm{O}$ artigo propõe um esquema de proteção do tipo MPP que permite o particionamento de $B_{t}$ de forma assimétrica entre as rotas utilizadas por cada par origem-destino $(o-d)$, de forma a encontrar partições que resultem em acréscimo ao tráfego total estabelecido, mas com menores probabilidades de bloqueio e um menor valor médio de banda espremida. Para isso, é proposta uma meta-heurística baseada em algoritmo genético para definir as parcelas de taxas de transmissão alocadas para cada rota disjunta entre cada par $o-d$ que promova redução tanto na probabilidade de bloqueio (PB) quanto no squeezing médio da rede. Um cenário de proteção contra falha de enlace simples (única) é considerado. Os resultados obtidos mostram que o esquema proposto é capaz de promover a redução do squeezing médio e da probabilidade de bloqueio da rede em relação ao esquema com partição igualitária do tráfego (PDPP).

\section{Provisionamento Multicaminho E Meta-Heuristica Proposta}

A utilização de MPP juntamente com o conceito de squeezing para proteção dedicada em redes ópticas elásticas resulta numa menor utilização de recursos e, consequentemente, um melhor desempenho da rede (em termos de $\mathrm{PB}$ ) em relação ao SPP, conforme mostrado em [6]. A Fig. 1 compara a $B_{t}$ dos esquemas DPP e PDPP, bem como os esquemas DPPS e PDPPS (DPP e PDPP que admitem squeezing), por meio de um exemplo de conexão entre os nós 0 e 2 . No exemplo, a $B_{r}$ da conexão é de $100 \mathrm{~Gb} / \mathrm{s}$. A Fig. 1a apresenta o estabelecimento da conexão utilizando o esquema DPP. A linha contínua na cor azul representa a rota de trabalho enquanto a linha tracejada representa a rota de proteção. O DPP utiliza $B_{r}=100 \mathrm{~Gb} / \mathrm{s}$ na rota de trabalho e $B_{i}=$ $100 \mathrm{~Gb} / \mathrm{s}$ na rota de proteção, resultando em $B_{t}=200 \mathrm{~Gb} / \mathrm{s}$. Por outro lado, o PDPP (exemplo da Fig. 1b) particiona a $B_{t}$ igualitariamente em $3 B_{s}$ e as transmite por 3 rotas disjuntas. Dessa forma, a soma de quaisquer duas $B_{s}$ sempre resulta na taxa de transmissão requisitada $B_{r}$, garantindo a proteção sem squeezing. No exemplo da Fig. $1 \mathrm{~b}, B_{s}=50 \mathrm{~Gb} / \mathrm{s}$, resultando numa $B_{t}=150 \mathrm{~Gb} / \mathrm{s}$, ou seja, uma redução de $25 \%$ na $B_{t}$ alocada em relação ao DPP. Note que, no caso de falha única de algum dos enlaces pertencentes a uma das 3 rotas, a proteção sem squeezing é garantida, pois a taxa resultante da soma das taxas das duas rotas remanescentes é sempre igual a $100 \mathrm{~Gb} / \mathrm{s}$.

Para avaliar o uso do provisionamento multicaminho com possibilidade de realizar squeezing na chamada, Assis et al. [5] apresentam dois parâmetros que podem ser usados para essa finalidade.

O primeiro é chamado de fator de compressão $(\beta)$, e corresponde ao valor percentual máximo aceitável de redução da taxa de transmissão (squeezing percentual máximo) na ocorrência de uma falha simples de enlace. Em caso de falha, $B_{r}$ pode ser totalmente ou parcialmente protegida, essa parcela de taxa de transmissão protegida é chamada de $B_{p}$. Pode-se calcular o parâmetro $\beta$ por

$$
\beta=\frac{B_{r}-B_{p}}{B_{r}} .
$$

O segundo é o parâmetro de incremento de taxa de transmissão $(\alpha)$, que pode ser calculado por

$$
\alpha=\frac{B_{t}-B_{r}}{B_{r}}=\frac{B_{t}}{B_{r}}-1 .
$$

As Figs. 1c e 1d mostram um exemplo de estabelecimento de uma conexão usando as estratégias DPPS e PDPPS, respectivamente, admitindo-se para ambos um squeezing máximo de $20 \%(\beta=0,2)$. O DPPS estabelece apenas a rota de proteção considerando tal redução, levando a $B_{t}=180 \mathrm{~Gb} / \mathrm{s}$ e $\alpha=0,8$. $\mathrm{O}$ valor da $B_{s}$ utilizada nos esquemas PDPP e PDPPS pode ser calculada por

$$
B_{s}=\frac{(1-\beta) \cdot B_{r}}{G-1},
$$

em que $G$ é o número de rotas utilizadas pelo esquema de proteção. No exemplo mostrado (Fig. 1d), o PDPPS beneficiase do particionamento do tráfego entre as 3 rotas e da banda espremida para operar com uma $B_{s}=40 \mathrm{~Gb} / \mathrm{s}$ em cada rota $\left(B_{t}=120 \mathrm{~Gb} / \mathrm{s}\right.$ e $\left.\alpha=0,2\right)$. Note que, nos dois casos, a $B_{p}=80 \mathrm{~Gb} / \mathrm{s}$ é mantida sob falha única. Entretanto, o PDPPS utiliza menos recursos em relação ao DPPS, assim como o PDPP em relação ao DPP. No caso do PDPP, note que tanto o tráfego de trabalho quanto o de proteção são roteados por todas as rotas estabelecidas. Por isso, neste trabalho, elas são chamadas indistintamente como rotas de trabalho.

Neste artigo, também é considerada a possibilidade de o PDPP operar com 2 rotas $(G=2)$, sendo a $B_{s}$ calculada usando a Eq. 3. Por isso, neste artigo, o PDPP com 2 rotas é chamado de PDPP2, enquanto que o PDPP de 3 rotas é chamado de PDPP3. Note que uma requisição de tráfego de $100 \mathrm{~Gb} / \mathrm{s}$ é estabelecida pelo PDPP2 da mesma forma que no DPP, pois, aplicando $\beta=0$ e $G=2$ na Eq. 3, tem-se $B_{s}=B_{r}$, ou seja, $B_{t}=200 \mathrm{~Gb} / \mathrm{s}$. Já aplicando o esquema PDPP2S, tem-se $\beta=0,2$ e a mesma conexão resulta numa $B_{s}=80 \mathrm{~Gb} / \mathrm{s}$ e $B_{t}=160 \mathrm{~Gb} / \mathrm{s}$.

\section{A. Proteção multicaminho com otimização de particiona- mento}

Embora a aplicação do esquema PDPP3 apresente baixo consumo de recursos com um particionamento simétrico de $B_{t}$ nas 3 rotas de trabalho, realizar tal particionamento de forma assimétrica oferece algumas oportunidades de otimização que impactam no desempenho da rede.

Há a necessidade de se introduzir um novo parâmetro de avaliação ao se assumir um particionamento assimétrico. Esse parâmetro é o beta médio $\left(\beta_{M}\right)$ da conexão, que corresponde 


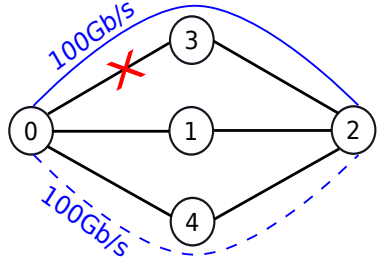

(a)

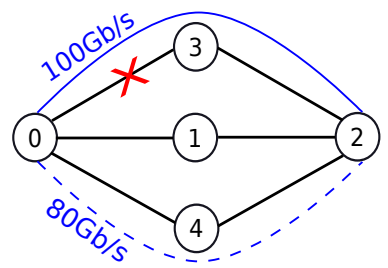

(c)

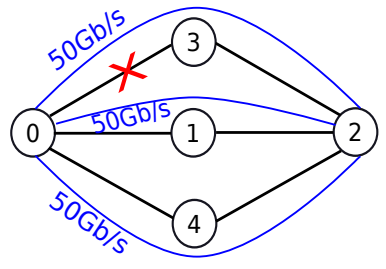

(b)

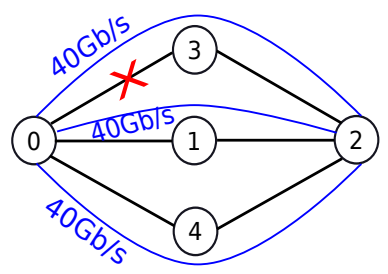

(d)
Fig. 1: Exemplo de estabelecimento de uma conexão de $100 \mathrm{~Gb} / \mathrm{s}$ entre os nós 0 e 2 pelos esquemas: (a) DPP $(\beta=0)$, (b) PDPP $(\beta=0)$, (c) DPPS $(\beta=0,2)$ e (d) PDPPS $(\beta=0,2)$.

ao valor esperado de $\beta$ condicionado à existência de uma falha em uma das rotas de trabalho da conexão. Isso é dado pelo somatório dos produtos dos betas resultantes $\beta^{\prime}$ da queda de cada uma das rotas pelas suas respectivas probabilidades de falha $(P)$, ou seja

$$
\beta_{M}=\sum_{n=1}^{G} \beta^{\prime}(n) \cdot P(n),
$$

em que $G$ é o número de rotas utilizadas pelo esquema de proteção, $\beta^{\prime}(n)$ é o beta resultante devido à falha da rota $n$ e $P(n)$ é a probabilidade de uma falha ocorrer na rota $n$. Considerando um cenário no qual todos os enlaces têm a mesma probabilidade de falha, o cálculo de $P(n)$ pode ser feito de forma simplificada. Seja $L_{n}$ o número de enlaces da rota $n$ e $L_{t}$ o número total de enlaces das $G$ rotas, tem-se

$$
P(n)=\frac{L_{n}}{L_{t}} .
$$

A Fig. 2 mostra um cenário de estabelecimento de uma conexão de $B_{r}=100 \mathrm{~Gb} / \mathrm{s}$ entre os nós 0 e $6, \operatorname{com} \beta=0,25$, largura de banda dos slots de $12,5 \mathrm{GHz}$, formato de modulação QAM-4, utilizando o particionamento simétrico proposto pelo esquema PDPP3 (Fig. 2a) e um particionamento assimétrico proposto neste artigo (Fig. 2b).

Fazendo uma análise em termos de $\beta_{M}$ nos exemplos mostrados na figura, tem-se que o $\beta_{M}$ da conexão da Fig $2 \mathrm{a}$ corresponde a $\beta_{M}=\left(0,25 \times \frac{2}{8}\right)+\left(0,25 \times \frac{2}{8}\right)+\left(0,25 \times \frac{4}{8}\right)=$ 0,25 , uma vez que uma falha única de enlace em qualquer uma das 3 rotas resulta sempre numa taxa de transmissão protegida $B_{p}=75 \mathrm{~Gb} / \mathrm{s}$.

Por outro lado, considerando o estabelecimento da conexão mostrada na Fig. 2 b, tem-se que $\beta_{M}=\left(0,25 \times \frac{2}{8}\right)+(0,25 \times$ $\left.\frac{2}{8}\right)+\left(0 \times \frac{4}{8}\right)=0,125$. Note que o valor da última parcela do somatório é nula, pois uma falha na rota $r_{3}$ não resulta em compressão de $B_{r}$, uma vez que a soma das $B_{s}$ das rotas remanescentes $\left(r_{1}\right.$ e $\left.r_{2}\right)$ é de $100 \mathrm{~Gb} / \mathrm{s}$, o que corresponde à própria $B_{r}$.

Verifica-se que $\beta_{M}$ do cenário da Fig. $2 \mathrm{~b}$ é menor que o $\beta_{M}$ do cenário da Fig. 2 a, isso implica num menor valor esperado largura de banda espremida em caso de falha na conexão do cenário mostrado na Fig. 2b. Uma análise semelhante pode ser feita em termos de $\alpha$. No cenário da Fig. 2a, $\alpha=0,125$, enquanto que, no cenário da Fig. $2 b, \alpha=0,25$.

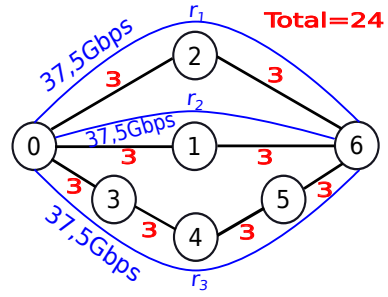

(a)

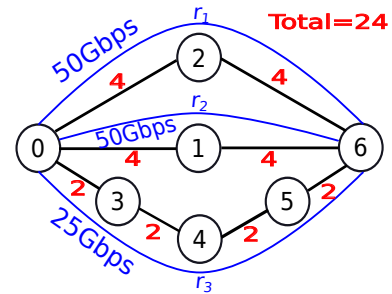

(b)
Fig. 2: Exemplo de uso de slots $(12,5 G H z)$ numa conexão de $100 \mathrm{~Gb} / \mathrm{s}$ estabelecida entre os nós 0 e 6 , com $\beta=$ 0,25 , formato de modulação QAM-4 e proteção multicaminho utilizando: (a) particionamento simétrico e (b) particionamento assimétrico.

A figura 2 também mostra a utilização de slots pelos esquemas simétrico e assimétrico (mostrada em vermelho ao lado de cada enlace). Analisando a utilização de slots no cenário da Fig. 2a, cada rota utiliza $B_{s}=37,5 \mathrm{~Gb} / \mathrm{s}$, que resulta na utilização de 3 slots por enlace. O número de enlaces usados pelas 3 rotas é igual a 8 , logo, há um consumo total de 24 slots. Por outro lado, no cenário da Fig. 2b, as rotas $r_{1}$ e $r_{2}$ utilizam $B_{s}=50 \mathrm{~Gb} / \mathrm{s}$, que exige o uso de 4 slots por enlace. A rota $r_{3}$ utiliza $B_{s}=25 \mathrm{~Gb} / \mathrm{s}$ e usa 2 slots por enlace. Assim, o total de slots utilizados é $(4 \times 4)+(2 \times 4)=24$. Pode-se concluir nessa análise que, em relação ao particionamento simétrico do cenário da Fig. 2a, o cenário de particionamento assimétrico proposto na Fig. $2 \mathrm{~b}$ oferece o benefício de menor $\beta_{M}$ para um mesmo consumo de recursos da rede. Adicionalmente, o particionamento assimétrico pode contribuir para a redução da probabilidade de bloqueio, uma vez que as $B_{s}$ que exigem a utilização de menos slots podem ser mais facilmente alocadas nas rotas mais longas, uma vez que essas últimas geralmente apresentam menores disponibilidades de slots livres e maiores interferências de outras rotas.

\section{Esquema de Proteção Proposto e Algoritmo GENÉTICO APLICADO AO PARTICIONAMENTO}

$\mathrm{O}$ esquema de proteção proposto neste trabalho visa realizar um adequado particionamento da $B_{t}$ entre as rotas de trabalho para cada par $o-d$ com o objetivo de reduzir, simultaneamente, a $P B$ e o $\beta_{M}$. Por isso, o esquema recebe o nome de OPDPP (Optimized Partitioning Dedicated Path Protection). Seu principal objetivo é encontrar e atribuir uma parcela adequada de taxa de transmissão $B_{s}$ para cada rota utilizada pelo esquema de proteção, de forma a obter-se as possibilidades de otimização apresentadas na Seção II. O problema apresenta um grande número possibilidades de valores discretos para a otimização, por isso foi utilizado um algoritmo genético (Genetic Algorithm - GA) baseado no 
NSGA-II [7] utilizado em diversos trabalhos de otimização em redes ópticas encontrados na literatura [8], [9], [10], [11].

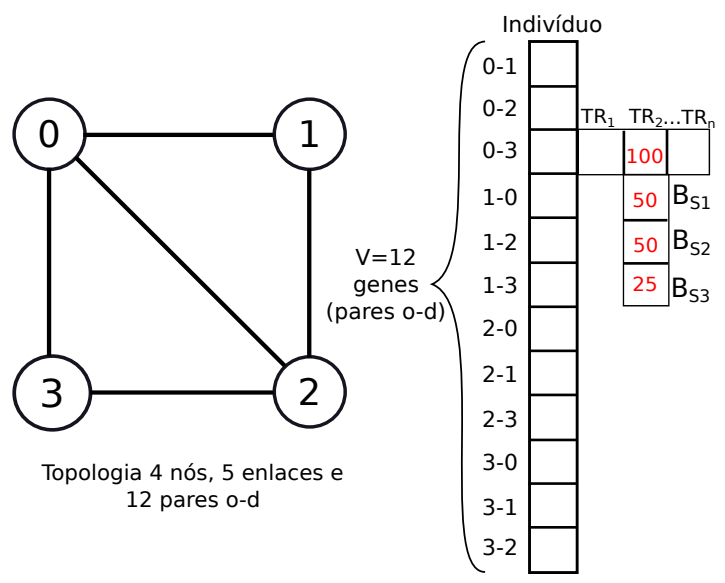

Fig. 3: Exemplo de representação do problema numa rede de 4 nós. Um indivíduo é representado por um vetor de 12 posições que contêm as $B_{s}$ para cada uma das rotas usadas pelo esquema de proteção para cada par origem-destino.

Assume-se que há um conjunto de taxas de transmissão que podem ser requisitadas pelas chamadas: $T R_{1}, T R_{2}, \ldots, T R_{n}$. Para cada taxa $T R_{i}$ há um conjunto $J$ de opções de escolhas assimétricas para a $B_{s}$ de cada uma das três rotas consideradas pelo algoritmo genético de forma que $\alpha_{\max }=0,4$ (i.e. o algoritmo genético proposto aqui assume o PDPP3S).

Cada indivíduo corresponde a uma lista que representa todos os pares origem-destino da rede, cujos genes correspondem aos valores de taxa de transmissão parcial $B_{s}$, para cada opção de tráfego demandado. Esses indivíduos são representados por meio de um tensor tridimensional. A primeira dimensão tem tamanho $V$, em que $V$ corresponde ao número de pares origem-destino; a segunda corresponde às opções de tráfego $\left(T R_{i}\right)$ disponíveis para as requisições de conexão; e a terceira dimensão contêm as prováveis distribuições de $B_{s}$. A Fig. 3 mostra um exemplo da representação do indivíduo.

$\mathrm{O}$ algoritmo inicia criando uma população inicial de indivíduos que, a cada iteração do algoritmo, são recombinados de maneira inspirada na mutação e cruzamento genético. A avaliação de qualidade de cada individuo é feita usando as funções objetivo $P B$ (probabilidade de bloqueio da rede) e $\beta_{M R}$ (beta médio da rede), que é dado por

$$
\beta_{M R}=\frac{\sum_{n=1}^{N_{p}} \beta_{M n}}{N_{p}},
$$

onde $N_{p}$ corresponde ao número de conexões protegidas na rede.

O algoritmo utiliza os valores de avaliação de cada indivíduo (fitness) para criar um conjunto de soluções não dominadas, denominado de frente de Pareto. Ao fim das iterações, a primeira frente de Pareto representa um conjunto dos melhores indivíduos (soluções) que realizam o melhor trade-off entre os dois objetivos considerados.

Ao final, o algoritmo entrega o indivíduo (solução) com o menor valor de $P B$ da primeira frente de Pareto. Esse indivíduo contém a distribuição de $B_{s}$ entre as rotas para cada demanda de tráfego e para cada par $o-d$ que resulta no melhor desempenho da rede em termos de $P B$ com a menor $B_{M R}$ encontrada para essa $P B$. O descrito até aqui nesta seção consiste na fase de otimização. Ela pode acontecer antes da rede entrar em operação.

Durante a fase de operação, por outro lado, as requisições são dinamicamente solicitadas à rede e o algoritmo de roteamento e alocação de espectro (Routing and spectrum assignment - RSA) juntamente com o controle de admissão de chamadas da rede utilizam as informações das $B_{s} \mathrm{~s}$ (para cada par $o-d$ e taxa $T R_{i}$ ) otimizadas obtidas durante a fase de otimização.

O OPDPP sempre tenta realizar a alocação da requisição utilizando o PDPP3S com as $B_{s}$ encontradas pelo GA. Caso não haja recursos disponíveis para atender a demanda da requisição nas 3 rotas, o algoritmo tenta realizar a alocação com o PDPP2S. Persistindo a indisponibilidade de recursos necessários para a alocação, a chamada é bloqueada.

\section{Simulações E Resultados}

Um simulador de eventos discretos capaz de simular probabilidade de bloqueio em redes ópticas elástica sob tráfego dinâmico, apresentado por Cavalcante et. al. [12], foi adaptado para simular os esquemas de proteção DPP e PDPP, bem como todas as métricas citadas neste trabalho. A Fig.4 apresenta a topologia NSFNET composta por 14 nós e 21 enlaces bidirecionais que foi utilizada nas simulações. A topologia EON-RT também foi simulada e obtidos resultados similares aos da NSFNET. As simulações foram configuradas para 128 slots por enlace, $10^{6}$ requisições, tráfego dinâmico com chegadas Poissonianas e distribuição Exponencial para a duração das chamadas. As opções de $B_{r}$ para as requisições de conexão são de 100,200 e $400 \mathrm{~Gb} / \mathrm{s}$, uniformemente distribuídas, $\beta=0,2$ e não foi considerado RMLSA. Foram utilizadas 50 gerações de 50 indivíduos para a simulação do Algoritmo Genético.

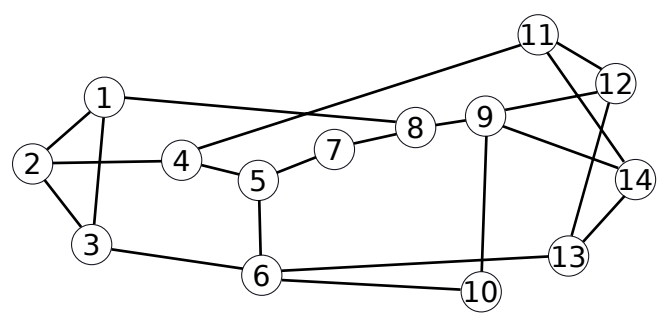

Fig. 4: topologia NSFNET.

O parâmetro $\alpha_{M R}$ representa o valor médio dos $\alpha$ de todas as conexões protegidas na rede, sendo definido por

$$
\alpha_{M R}=\frac{\sum_{n=1}^{N_{p}} \alpha_{n}}{N_{p}} .
$$

A Fig. 5 mostra as curvas obtidas por cada esquema de proteção em termos de $\mathrm{PB}$ em função da carga aplicada. Analisando os esquemas que não admitem squeezing, observase que as curvas do DPP (preta) e PDPP2 (amarela) estão sobrepostas, pois os dois esquemas alocam as requisições da mesma maneira, enquanto a curva do PDPP3 (ciano) apresenta os menores valores deste grupo. 


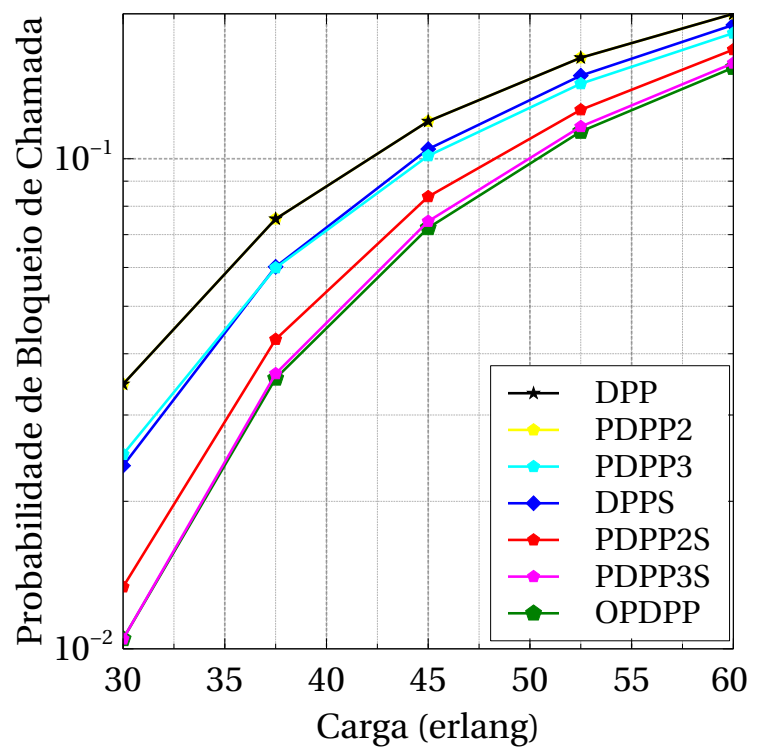

Fig. 5: Probabilidade de bloqueio de chamada em função da carga da rede para a topologia NSFNET.

Entre os esquemas que admitem squeezing e usam particionamento simétrico de $B_{r}$, o esquema PDPP3S (curva magenta) apresentou o melhor desempenho, seguido do PDPP2S (curva vermelha) e do DPPS (curva azul). O esquema OPDPP, com particionamento assimétrico otimizado pelo GA, obteve um resultado ligeiramente melhor que o PDPP3S, conforme mostrado na Fig. 5. No entanto, alocar as conexões usando o OPDPP resultou em uma redução no $\beta_{M}$ das conexões em relação ao PDPP3S. Essa análise pode ser vista no gráfico mostrado na Fig 6, na qual o OPDPP apresenta $\beta_{M R}=0,161$ e o PDPP3S $\beta_{M R}=0,2$. Ou seja, apesar de os dois esquemas apresentarem probabilidades de bloqueio semelhantes, na ocorrência de uma falha de enlace a redução média da taxa de transmissão promovida pelo OPDPP é menor do que a promovida pelo PDPP3S (16,1\% contra $20 \%$ respectivamente). Isso mostra que particionamento assimétrico inteligente pode melhorar o compromisso entre resiliência e taxa de transmissão disponível na rede.

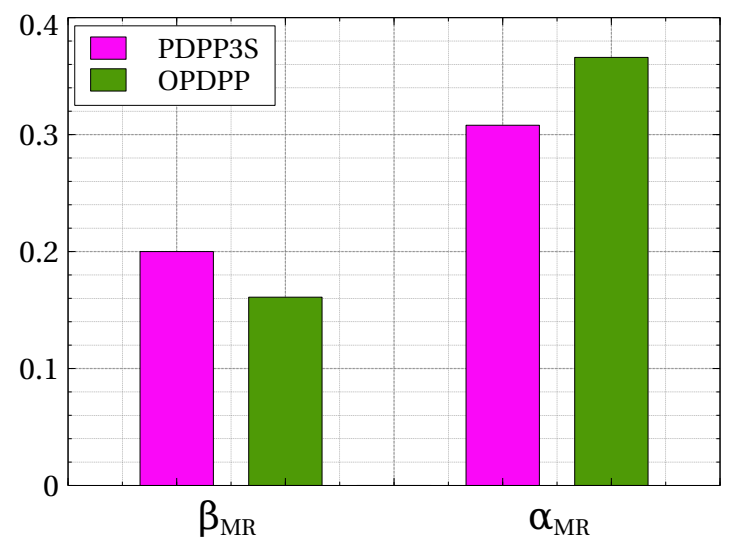

Fig. 6: Resultados obtidos pelos esquemas PDPP3S e OPDPP em termos de $\beta_{M R}$ e $\alpha_{M R}$.

Por outro lado, o OPDPP promove um consumo de recursos da rede ligeiramente superior ao do PDPP3S, como pode ser visto analisando os valores de $\alpha_{M R}$ obtidos pelos algoritmos e mostrados na Fig 6. No entanto, isso não afeta os principais parâmetros de desempenho da rede (PB e $\beta_{M R}$ ).

\section{CONCLUSÕES}

Este artigo apresenta uma abordagem de proteção dedicada com provisionamento multicaminho assimétrico para redes elásticas de tráfego dinâmico. Um novo parâmetro chamado squeezing médio $\left(\beta_{M}\right)$ é proposto e utilizado para avaliação da eficiência na alocação de tráfego de esquemas de proteção multicaminho. Este trabalho discute que o uso de taxas de transmissão distribuídas de forma assimétrica entre as rotas do esquema de proteção PDPP pode resultar em redução da PB e do $\beta_{M}$ da rede. É apresentado um novo esquema otimizado de proteção chamado de OPDPP que utiliza algoritmo genético multiobjetivo para encontrar e atribuir, a cada rota utilizada entre os pares origem-destino, uma taxa de transmissão parcial que promova a redução da $\mathrm{PB}$ e do $\beta_{M R}$. Os resultados obtidos nas simulações mostraram que, no cenário investigado, o OPDPP apresentou resultados ligeiramente melhores de PB e também um melhor $\beta_{M R}$ em relação aos outros algoritmos considerados neste trabalho.

\section{REFERÊNCIAS}

[1] K. Takeda, T. Sato, R. Shinkuma, and E. Oki, "Multipath provisioning scheme for fault tolerance to minimize required spectrum resources in elastic optical networks," Computer Networks, vol. 188, p. 107895, 2021.

[2] S. Paira, J. Halder, U. Bhattacharya, and M. Chatterjee, "A novel fragmentation-aware and energy-efficient multipath routing and spectrum allocation for prioritized traffic in protected eons," in 2020 11th International Conference on Computing, Communication and Networking Technologies (ICCCNT). IEEE, 2020.

[3] F. Paolucci, A. Castro, F. Cugini, L. Velasco, and P. Castoldi, "Multipath restoration and bitrate squeezing in sdn-based elastic optical networks," Photonic network communications, vol. 28, no. 1, pp. 45-57, 2014.

[4] K. D. Assis, R. C. Almeida, and H. Waldman, "Milp formulation for squeezed protection in spectrum-sliced elastic optical path networks," in 2012 International Symposium on Performance Evaluation of Computer \& Telecommunication Systems (SPECTS). IEEE, 2012.

[5] K. Assis, S. Peng, R. Almeida, H. Waldman, A. Hammad, A. Santos, and D. Simeonidou, "Network virtualization over elastic optical networks with different protection schemes," Journal of Optical Communications and Networking, vol. 8, no. 4, pp. 272-281, 2016.

[6] N. Shahriar, S. Taeb, S. R. Chowdhury, M. Zulfiqar, M. Tornatore, R. Boutaba, J. Mitra, and M. Hemmati, "Reliable slicing of 5g transport networks with dedicated protection," in 2019 15th International Conference on Network and Service Management (CNSM). IEEE, 2019.

[7] K. Deb, A. Pratap, S. Agarwal, and T. Meyarivan, "A fast and elitist multiobjective genetic algorithm: Nsga-ii," IEEE transactions on evolutionary computation, vol. 6, no. 2, pp. 182-197, 2002.

[8] M. A. Cavalcante, H. A. Pereira, D. A. Chaves, and R. C. Almeida, "Evolutionary multiobjective strategy for regenerator placement in elastic optical networks," IEEE Transactions on Communications, vol. 66 , no. 8, pp. 3583-3596, 2018.

[9] B. V. Correia, R. C. Almeida, D. A. Chaves, and H. A. Pereira, "Optical inverse multiplexing technique applied to elastic optical networks," in 2019 21st International Conference on Transparent Optical Networks (ICTON). IEEE, 2019.

[10] D. P. Pinto Roa and R. Lugo, "Sub-graph based multicast protection in wdm networks: A multi/many-objective evolutionary algorithms approaches," in XXIV Congreso Argentino de Ciencias de la Computación (La Plata, 2018)., 2018.

[11] A. Eira, J. Santos, J. Pedro, and J. Pires, "Multi-objective design of survivable flexible-grid dwdm networks," Journal of Optical Communications and Networking, vol. 6, no. 3, pp. 326-339, 2014.

[12] M. A. Cavalcante, H. A. Pereira, and R. C. Almeida, "Simeon: an opensource elastic optical network simulator for academic and industrial purposes," Photonic Network Communications, vol. 34, no. 2, pp. 193 201, 2017 